\title{
DOENÇAS TROPICAIS NEGLIGENCIADAS: UMA NOVA ERA DE DESAFIOS E OPORTUNIDADES
}

\section{Luiz C. Dias" e Marco A. Dessoy}

Departamento de Química Orgânica, Instituto de Química, Universidade Estadual de Campinas, Cidade Universitária Zeferino Vaz, CP 6154, 13083-970 Campinas - SP, Brasil

Rafael V. C. Guido, Glaucius Oliva e Adriano D. Andricopulo*

Instituto de Física de São Carlos, Universidade de São Paulo, Av. Trabalhador São-Carlense, 400, 13560-970 São Carlos - SP, Brasil

Recebido em 18/9/13; aceito em 20/10/13; publicado na web em 01/11/2013

NEGLECTED TROPICAL DISEASES: A NEW ERA OF CHALLENGES AND OPPORTUNITIES. In an article recently published in Química Nova, entitled "Chemistry Without Borders" (“Química Sem Fronteiras") [Pinto, A. C.; Zucco, C.; Galembeck, F.; Andrade, J. B.; Vieira, P. C. Quim. Nova 2012, 35, 2092], the authors highlighted the important aspects of science and technology with special emphasis on the field of Chemistry and its contributions toward a more prosperous Brazil of future. As a second step in that direction, this article extends the discussion of a key issue for the country in the framework of the chemistry community through the so called position papers in strategic areas. This document is a part of the contribution of the Brazilian Chemical Society to the World Science Forum to be held in Rio de Janeiro in November 2013. In this context, the present paper provides a brief discussion on neglected tropical diseases (NTDs) with emphasis on the current challenges and opportunities towards the development and evolution of the field. NTDs leads to illness, long-term disability or death, and has severe social, economic and psychological consequences for millions of men, women, and children worldwide. In most cases, the available treatments are inadequate and extremely limited in terms of efficacy and safety, leading to an urgent demand for new drugs. In addition to the traditional challenges involved in any drug discovery process, it is widely recognized that there is an innovation gap and a lack of investment for research and development (R\&D) in the area of NTDs. In the last few decades, methods toward combating, eradication, prevention, and treatment of NTDs have been repeatedly emphasized in the major international agendas. Developments in these strategies and alliances have continued to have an essential impact, particularly in the area of drug discovery, both in Brazil and globally and should be encouraged and supported. Several examples of international activities dedicated to the reduction of the devastating global impact of NTDs can be provided. Despite the beneficial developments in the past 30 years, NTDs continue to devastate poor communities in remote and vulnerable areas, in large part, due to market failures and public policies. Recent studies have shown that among 756 new drugs approved between 2000 and 2011, only four new chemical entities (NCEs) were identified for the treatment of malaria, while none were developed against NTDs or tuberculosis. Furthermore, only $1.4 \%$ of approximately 150,000 clinical trials were registered for neglected diseases, with a smaller number of trials for NCEs. Establishment and strengthening of global strategies involving the triad "government-academia-industry" is fundamental to the success in R\&D of new drugs for NTDs. National and international public-private initiatives that aim to create, encourage, and invest in $R \& D$ projects have been implemented and therefore are of utmost importance to successfully integrate Brazil into this new paradigm. It is essential to lay the foundation for mechanisms that will intensify investments in infrastructure, training, and qualification of personnel with an ultimate strategic vision that foresees continuity. Our research group has made significant contributions to the development of this field with the goal of forging new frontiers while tackling both current and future challenges that include indispensable elements such as innovation and integration.

Keywords: neglected tropical diseases; drug discovery; medicinal chemistry

\section{INTRODUÇÃO}

Em um artigo publicado recentemente em Química Nova,${ }^{1}$ intitulado "Química Sem Fronteiras", os autores destacaram importantes aspectos de C\&T (Ciência e Tecnologia) com especial ênfase para a área de Química e para a sua contribuição para um futuro mais próspero, seguro e justo para a nação. Em uma segunda etapa, esta ação se estende ao debate de questões estratégicas para o país através da contribuição da comunidade Química com chamados position papers em áreas estratégicas. Este documento será parte da contribuição da Sociedade Brasileira de Química (SBQ) ao Fórum Mundial de Ciências, que acontecerá no Rio de Janeiro em novembro de 2013. Neste contexto, o presente artigo colabora com uma breve abordagem sobre as doenças tropicais negligenciadas, que se caracterizam como

*e-mail: aandrico@ifsc.usp.br

\#e-mail: 1dias@iqm.unicamp.br uma área estratégica de C\&T para o país em um tópico de extremo interesse para a nossa sociedade, como a saúde humana. O texto destaca os desafios e oportunidades relacionados à descoberta de novos fármacos para uma diversidade de doenças que afetam milhões de pessoas e representam uma crescente ameaça mundial.

\section{DOENÇAS TROPICAIS NEGLIGENCIADAS}

As doenças tropicais negligenciadas (DTNs) afetam principalmente, mas não exclusivamente, populações pobres nas regiões mais vulneráveis e miseráveis do planeta. De acordo com a Organização Mundial da Saúde (OMS), ${ }^{2}$ atualmente 17 doenças parasitárias crônicas são classificadas como DTNs: dengue, raiva, tracoma, úlcera de Buruli (infecção por Mycobacterium ulcerans), treponematoses endêmicas, hanseníase (doença de Hansen), doença de Chagas (tripanossomíase americana), doença do sono (tripanossomíase africana), leishmaniose, cisticercose, dracunculíase (infecção pelo 
verme da guiné), equinococose, infecções por trematódeos causadas por alimentos, filariose linfática, oncocercose (cegueira dos rios), esquistossomose (bilharziose) e helmintíases transmitidas pelo solo. Doenças negligenciadas é outro termo bastante empregado, mas devemos observar que se refere a todas as DTNs incluindo HIV/ AIDS, tuberculose, malária e outras infecções negligenciadas. As DTNs impõem limitações sérias às sociedades atingidas, levando a um panorama de enfermidade, sofrimento, incapacidade e morte, com graves consequências sociais, econômicas e psicológicas para milhões de homens, mulheres e crianças.

Já há muito tempo, mas principalmente na última década, estão em pauta nas principais agendas internacionais as formas de combate, erradicação, prevenção e tratamento das DTNs. Especialmente na área de novos fármacos, a criação e o desenvolvimento de estratégias e alianças são opções de admirável impacto tanto no Brasil quanto no exterior e devem ser incentivadas e apoiadas, pois somente desta maneira poderemos conquistar novos fronteiras capazes de satisfazer as exigências impostas por um mercado em contínua evolução.

\section{ESTRATÉGIAS E ALIANÇAS}

Vários exemplos podem ser citados para ilustrar ações internacionais dedicadas a reduzir o impacto global devastador das DTNs. ${ }^{3}$ Importantes alianças têm sido intensificadas entre a OMS e indústrias farmacêuticas, tais como: (i) a GlaxoSmithKline (GSK) com a doação de 400 milhões de comprimidos por ano (a partir de 2012) de albendazol para o tratamento de crianças com vermes intestinais, que vem a somar-se aos outros 600 milhões de comprimidos por ano de albendazol da GSK do Programa Global de Eliminação da Filariose Linfática, totalizando 1 bilhão de comprimidos; (ii) a Sanofi que renovou em março de 2011 o seu acordo para doação de quantidades ilimitadas de eflornitina, melarsoprol e pentamidina para o tratamento da tripanossomíase africana. A empresa apoia também o programa da OMS de controle da tripanossomíase africana, com um valor de US\$ 25 milhões por um período de 5 anos. Outros programas que recebem apoio são voltados para o tratamento da úlcera de Buruli, doença de Chagas, leishmaniose e bouba; (iii) a Bayer expandiu seu acordo com a OMS aumentando a sua doação de nifurtimox para 5 milhões de comprimidos até 2017 para o tratamento das tripanossomíases americana e africana. A empresa doa também quantidades ilimitadas de suramina para o tratamento da tripanossomíase africana, auxiliando também com o financiamento e a logística para a distribuição dos medicamentos; (iv) a Johnson \& Johnson está expandindo sua doação de 50 milhões de comprimidos de mebendazol para 200 milhões por ano; (v) a Novartis continuará a doação do tratamento quimioterápico (rifampicina, dapsona e clofazimina) para hanseníase até 2015, incorporando também a doação de triclabendazol para o tratamento da fasciolíase; (vi) a Pfizer continuará a doação de quantidades ilimitadas de azitromicina para o tratamento do tracoma; (vii) a Merck \& Co. fornecerá suprimentos ilimitados de ivermectina para as regiões afetadas pela filariose linfática e oncocercose; (viii) a Merck KGaA contribuirá para o controle da esquistossomose com a doação de 200 milhões de comprimidos de praziquantel ao ano, até 2017. Estes são dados significativos que mostram o papel essencial das alianças internacionais para o combate das DTNs em todo o mundo.

Outros importantes exemplos de alianças globais reúnem parceiros públicos e privados na luta contra as doenças mais devastadoras. ${ }^{4}$ Em 30 de janeiro de 2012, em uma reunião no Royal College of Physicians em Londres, a diretora geral da OMS, Margaret Chan, anunciou a aprovação da Declaração de Londres para erradicar, eliminar e intensificar o controle das principais DTNs até o final desta década. A publicação do chamado $W H O$ roadmap define ações ousadas para acabar com a miséria causada pelas DTNs. Nesta iniciativa, a OMS contará com o apoio dos governos dos Estados Unidos, Reino Unido e Emirados Árabes, além de várias grandes companhias farmacêuticas, da Fundação Bill \& Melinda Gates e do Banco Mundial. As principais medidas anunciadas incluem: (i) manutenção e expansão de programas de doação de medicamentos para suprir as demandas até 2020; (ii) fornecimento de cerca de US\$ 800 milhões para apoiar os programas de eliminação de doenças; (iii) implementação e fortalecimento de programas de distribuição de medicamentos; e (iv) incentivo a integração de esforços e a investigação de novos compostos para promover o desenvolvimento de novos medicamentos. Este último item (iv) é de especial interesse no contexto desta contribuição que tem como foco explorar os desafios e oportunidades na área de novos fármacos para as DTNs.

\section{A URGÊNCIA POR NOVOS MEDICAMENTOS}

As opções terapêuticas disponíveis atualmente para as DTNs são muito limitadas, insuficientes e apresentam uma série de problemas, tais como baixa eficácia, elevada toxicidade e a emergência de cepas resistentes. Este panorama é sensivelmente agravado pela falta de investimentos e inovação em programas de $\mathrm{P} \& \mathrm{D}$ (pesquisa e desenvolvimento) de fármacos, que reflete no número extremamente reduzido de medicamentos que chegaram ao mercado ao longo das últimas décadas. ${ }^{5}$

Em dezembro de 2012, um estudo realizado pela Iniciativa de Medicamentos para Doenças Negligenciadas (DNDi, da sigla inglesa para Drugs for Neglected Diseases Initiative) e pela organização Médicos Sem Fronteiras (Doctors Without Borders/Médecins Sans Frontières, MSF) sobre a evolução das ações de $\mathrm{P} \& \mathrm{D}$ para doenças negligenciadas revelou que dos 756 novos fármacos aprovados entre 2000 e 2011, 29 (3,8\%) foram indicados para doenças negligenciadas, ainda que a carga global dessas doenças seja superior a $10 \%$. Além disso, apenas quatro eram novas entidades químicas (NCEs, da sigla inglesa para new chemical entities), das quais três foram indicadas para o tratamento da malária e nenhuma para tuberculose ou qualquer das DTNs. ${ }^{6}$ Além disso, apenas $1,4 \%$ de um total de cerca de 150 mil ensaios clínicos registrados se concentraram em doenças negligenciadas, com um número muito reduzido de ensaios para NCEs.

O progresso científico e tecnológico dos últimos 30 anos gerou inovações terapêuticas notáveis e um ganho substancial na expectativa e qualidade de vida das populações do mundo todo. Por outro lado, as DTNs continuam a devastar comunidades pobres nas regiões mais remotas e vulneráveis, devido, em grande parte, a falhas de mercado e de políticas públicas. Portanto, o estabelecimento e fortalecimento de estratégias empreendendo a tríade "governo - academia -indústria" são fundamentais para o sucesso de ações na área de $\mathrm{P} \& \mathrm{D}$ de fármacos para as DTNs. Alguns exemplos de parcerias público-privadas que tem como objetivo criar, estimular e investir em $\mathrm{P} \& \mathrm{D}$ de fármacos contra uma variedade de DTNs podem ser mencionados, como o Programa Especial para Pesquisa e Treinamento em Doenças Tropicais (TDR, da sigla inglesa para Special Programme for Research and Training in Tropical Diseases) da OMS, o Programa Medicamentos para Malária (MMV, da sigla inglesa para Medicines for Malaria Venture) e a Iniciativa de Medicamentos para Doenças Negligenciadas (DNDi).

\section{INICIATIVAS NACIONAIS DE P\&D}

Iniciativas de extrema importância vêm sendo executadas com sucesso para inserir o Brasil em um panorama cada vez mais expressivo no campo da $\mathrm{P} \& \mathrm{D}$ de fármacos. No entanto, em razão da falta de perspectivas de lucros na área de DTNs, os fatores que motivam o estabelecimento de parcerias são fundamentalmente diferentes daqueles aos que está habituada a indústria farmacêutica que procura fins 
lucrativos. Por exemplo, o recém-criado Centro de Pesquisa e Inovação em Biodiversidade e Fármacos (CIBFar), apoiado no programa CEPIDs (Centros de Pesquisa, Inovação e Difusão) da Fundação de Amparo à Pesquisa do Estado de São Paulo (FAPESP), é uma iniciativa resultante de projetos de pesquisa colaborativos, envolvendo: (i) Laboratório de Química Medicinal e Computacional (LQMC) e Laboratório de Biofísica Molecular - IFSC - USP, (ii) Núcleo de Bioensaios, Biossíntese e Ecofisiologia de Produtos Naturais (NUBBE) - IQ UNESP, (iii) Laboratórios de Síntese Orgânica - IQ - UNICAMP, (iv) Laboratórios de Produtos Naturais e Síntese Orgânica - DQ UFSCar, e (v) Laboratório de Produtos Naturais - FCFRP - USP. O CIBFar investe na realização de ciência básica e aplicada, bem como no desenvolvimento tecnológico em todas as áreas de biodiversidade e de descoberta de fármacos com base em pesquisas que utilizam o estado da arte da química de produtos naturais, química orgânica sintética, biologia molecular e estrutural, bioquímica, química medicinal, planejamento de fármacos e ensaios farmacológicos. O objetivo final é o desenvolvimento de NCEs com elevado potencial de inovação para desenvolvimento clínico, principalmente na área de DTNs. Para tanto, o CIBFar se baseia não apenas nas competências e conhecimentos científicos sólidos em todas as áreas de interesse, mas também em uma estrutura organizada para a integração das abordagens modernas em biodiversidade e descoberta de fármacos.

Outro exemplo é o Instituto Nacional de Ciência e Tecnologia de Biotecnologia Estrutural e Química Medicinal em Doenças Infecciosas (INBEQMeDI), que é uma iniciativa multidisciplinar com foco no desenvolvimento de candidatos a novos fármacos empregando alvos moleculares específicos em micro-organismos associados a doenças infecciosas. O INBEQMeDI foi criado no programa Institutos Nacionais de Ciência e Tecnologia (INCT) do MCT/CNPq/FAPESP. Este programa tem metas ambiciosas e abrangentes em termos nacionais como possibilidade de mobilizar e agregar, de forma articulada, os melhores grupos de pesquisa em áreas de fronteira da ciência e em áreas estratégicas para o desenvolvimento sustentável do país; impulsionar a pesquisa científica básica e fundamental competitiva internacionalmente; estimular o desenvolvimento de pesquisa científica e tecnológica de ponta associada a aplicações para promover a inovação e o espírito empreendedor, em estreita articulação com empresas inovadoras, entre outras. $\mathrm{O}$ INBEQMeDI tem como sede o Instituto de Física de São Carlos da USP. Fazem parte também os grupos associados: (i) Departamento de Química da Universidade Federal de São Carlos (DQ-UFSCar), (ii) Institutos de Biociências (IB) e de Ciências Biológicas (ICB) da USP, (iii) Faculdade de Medicina de Ribeirão Preto (FMRP) da USP, (iv) Faculdade de Ciências Farmacêuticas de Ribeirão Preto (FCFRP) da USP, (v) Instituto de Química da USP, (vi) Universidade Estadual de Ponta Grossa (UEPG) e, (vii) Universidade Federal de Viçosa (UFV). Outros INCTs com destacada atuação e grande experiência em P\&D de doenças negligenciadas são o IDN (Instituto Nacional de Ciência e Tecnologia de Inovação em Doenças Negligenciadas), que tem sua sede na Fundação Oswaldo Cruz (Fiocruz) no Rio de Janeiro, e o INOFAR (Instituto Nacional de Ciência e Tecnologia de Fármacos e Medicamentos), sediado no Centro de Ciências da Saúde (CCS), da Universidade Federal do Rio de Janeiro (UFRJ). Estes INCTs congregam pesquisadores renomados de diversos institutos e universidades e têm como objetivos principais promover a inovação em saúde e atuar na descoberta de novos fármacos para doenças negligenciadas consideradas prioridades para o país, bem como contribuir na formação e qualificação de profissionais da área, em vários níveis.

Vale também destacar que o grupo de autores do presente artigo foi selecionado pela Organização Mundial da Saúde em seu Programa Especial para Pesquisa e Treinamento em Doenças Tropicais (TDR,
OMS), como Centro de Referência Mundial em Química Medicinal e Síntese Orgânica para Doença de Chagas. O objetivo fundamental do grupo foi avançar no desenvolvimento de candidatos a novos fármacos para o tratamento da doença de Chagas. As atividades de pesquisa envolveram uma integração efetiva com laboratórios parceiros da rede de química medicinal da OMS, incluindo grandes empresas farmacêuticas como a Pfizer, Merck, Chemtura e Pharmacopeia. No momento, o grupo está envolvido em uma nova parceria internacional com a DNDi, visando o desenvolvimento de candidatos a novos fármacos para a doença de Chagas e leishmaniose. O modelo colaborativo da DNDi reúne diversos parceiros internacionais em interfaces complementares fundamentais, que compartilham os mesmos objetivos e levam adiante os projetos de P\&D. Uma nova parceria estabelecida recentemente com a MMV para o desenvolvimento de novos fármacos para a terapia da malária também merece destaque.

\section{DESCOBERTA DE NOVOS FÁRMACOS PARA AS DTNS: GRANDES DESAFIOS}

O processo de descoberta e desenvolvimento de fármacos é bastante complexo, tendo suas raízes profundamente ligadas às inovações científicas e tecnológicas. Este processo pode ser dividido em duas grandes fases: (i) descoberta e (ii) desenvolvimento, de acordo com a representação ilustrativa da Figura $1{ }^{7,8}$ Nos estágios iniciais do processo de descoberta, as pesquisas se concentram na identificação e otimização de moléculas pequenas capazes de modular a atividade do alvo macromolecular eleito para o processo de planejamento. A validação do alvo molecular selecionado é fundamental, pois estabelece a sua relevância no processo fisiopatológico em estudo, além de evidenciar se a sua modulação seletiva é capaz de gerar a resposta farmacológica esperada no controle da doença ou disfunção em humanos. ${ }^{9}$

As moléculas bioativas ou ligantes (do termo em inglês hits) têm sua origem a partir de produtos naturais ou sintéticos e podem ser identificadas por meio de triagens reais (in vitro) ou virtuais (in silico), ou ainda através de planejamento racional. Independente do modo usado para a identificação da substância química, a determinação e a validação experimental de suas propriedades biológicas são essenciais. Em geral, esta fase inicial de triagem leva a seleção de moléculas com baixa afinidade e que necessitam ser otimizadas em relação a uma série importante de propriedades (e.g., potência, afinidade, seletividade, absorção, toxicidade). Os compostos bioativos com melhores propriedades são eleitos como compostos líderes (do termo em inglês lead compounds) para etapas posteriores de otimização molecular. ${ }^{10}$

Com o auxílio de métodos de química medicinal é possível explorar o imenso espaço químico delineando o trabalho investigativo na identificação, seleção e otimização de moléculas capazes de interagir com alta afinidade e seletividade com o alvo molecular selecionado (e.g., enzima, receptor), o qual representa o espaço biológico do binômio químico-biológico em questão. Várias estratégias em química medicinal podem ser empregadas para a investigação do espaço químico-biológico, como a organização de bases padrões de dados, a aplicação de filtros moleculares, o emprego de ensaios biológicos automatizados em larga escala (HTS, da sigla inglesa para high-throughput screening), a triagem virtual (VS, da sigla inglesa para virtual screening), as técnicas de planejamento baseado na estrutura do receptor (SBDD, da sigla inglesa para structure-based drug design) e de planejamento baseado na estrutura do ligante (LBDD, da sigla inglesa para ligand-based drug design), além dos estudos das relações quantitativas entre a estrutura e atividade (QSAR, da sigla inglesa para quantitative structure-activity relationships). Em todos estes casos, o gerenciamento qualificado 


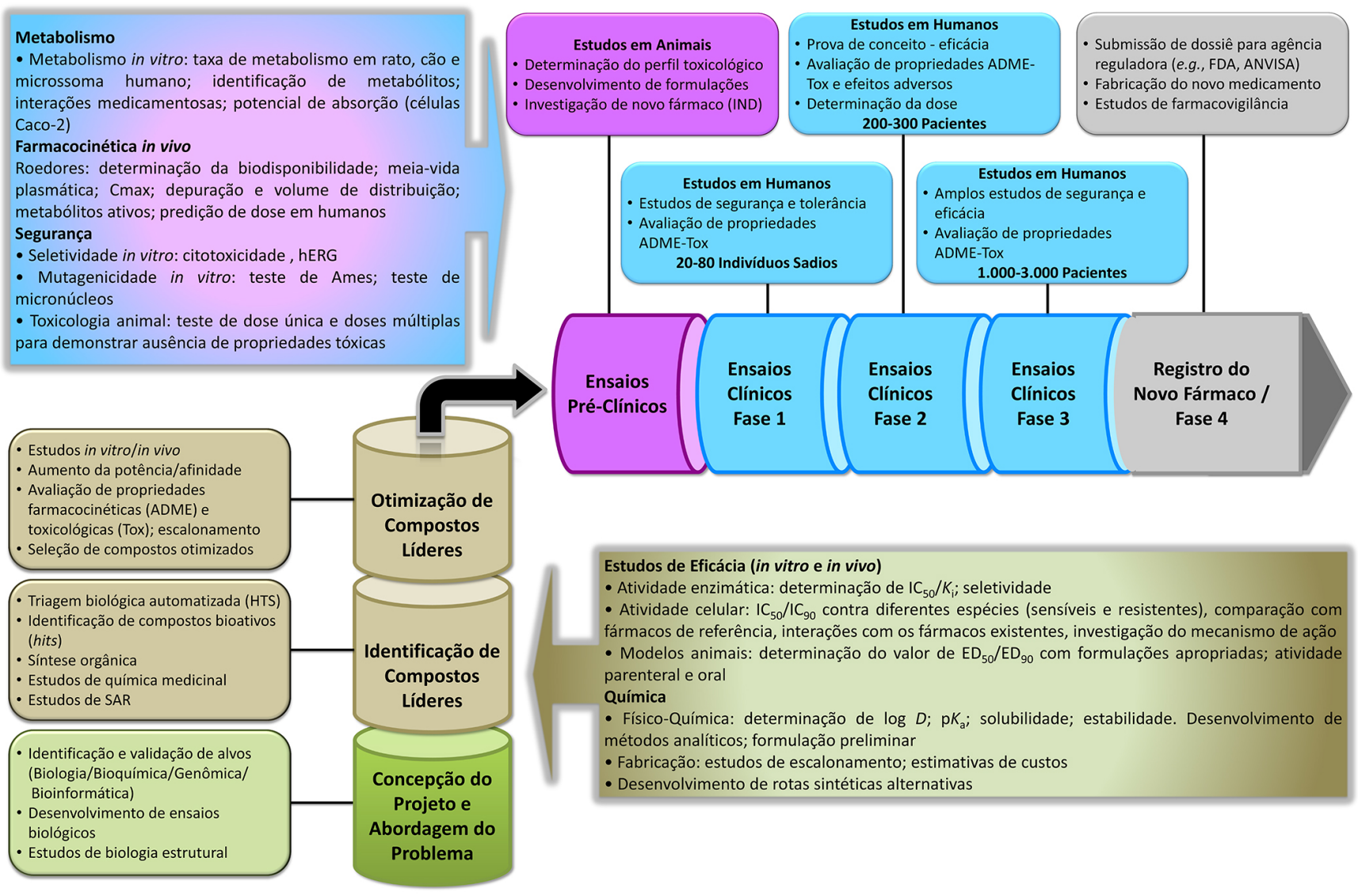

Figura 1. Etapas envolvidas no processo de descoberta e desenvolvimento de fármacos. Sendo, SAR, relações entre a estrutura e atividade; QSAR, relações quantitativas entre a estrutura e atividade; ADME/Tox, propriedades farmacocinéticas/toxicidade: Absorção, Distribuição, Metabolismo e Excreção

da informação possibilita a geração de conhecimento apropriado a partir dos dados disponíveis.

Uma das etapas-chave da Figura 1 é a otimização de compostos líderes em relação às suas propriedades farmacodinâmicas e farmacocinéticas, com o objetivo da descoberta de NCEs com elevado potencial de desenvolvimento clínico. Variações estruturais são utilizadas para adequar o perfil das moléculas às características necessárias ao uso terapêutico em humanos. Nesse sentido, a química medicinal oferece várias estratégias capazes de gerar coleções dirigidas de compostos. ${ }^{11,12}$ Os estudos das relações entre a estrutura e atividade (SAR, da sigla inglesa para structure-activity relationships) e de QSAR são importantes para guiar a síntese de novas moléculas com propriedades otimizadas, minimizando o universo de compostos a ser considerado nos dispendiosos programas de triagem biológica. Idealmente, propriedades farmacodinâmicas tais como potência, afinidade e seletividade, além de propriedades farmacocinéticas de ADME (Absorção, Distribuição, Metabolismo e Excreção), devem ser consideradas conjuntamente, facilitando a eliminação de candidatos com propriedades inadequadas e reduzindo os custos do processo de P\&D. Na fase de descoberta os estudos de pesquisa básica são essenciais. Nesta etapa é realizada uma série de testes in vitro e in vivo para avaliar o potencial dos candidatos a NCEs em relação a sua segurança e eficácia, visando a sua introdução em fases clínicas para testes em humanos.

Os métodos em quimio- e bioinformática estão cada vez mais integrados ao planejamento de fármacos, com notável e crescente relevância. O seu emprego nos estudos de química medicinal ocorre desde a identificação, seleção e otimização de moléculas candidatas, até a proposição de novas NCEs com elevado potencial terapêutico. A integração desses métodos ao trabalho químico e biológico experimental é um requerimento essencial para a geração de novas moléculas bioativas qualificadas, com propriedades múltiplas otimizadas. ${ }^{13}$

As NCEs selecionadas na fase de descoberta são investigadas em estudos pré-clínicos que visam, principalmente, avaliar a sua segurança e toxicidade em modelos animais padronizados e bem estabelecidos. Após a conclusão desta etapa, as NCEs mais promissoras são submetidas à aprovação das agências reguladoras, como a Administração Federal de Alimentos e Medicamentos dos Estados Unidos (FDA, da sigla inglesa para U.S. Food and Drug Administration) ou a Agência Nacional de Vigilância Sanitária (ANVISA), para autorização da realização de testes em humanos através da licença para investigação de um novo candidato a fármaco (IND, da sigla inglesa para investigational new drug) (Figura 1).

Nas fases clínicas de desenvolvimento são realizados diversos estudos para avaliar a eficácia e segurança das NCEs candidatas a fármacos. De forma resumida, na fase clínica I são realizados vários testes de dosagem dos princípios ativos em grupos de voluntários saudáveis (20-80 indivíduos) para avaliação da segurança. Na fase clínica II se iniciam os testes em indivíduos que apresentam a doença ou disfunção alvo (200-300 indivíduos), para a avaliação de parâmetros tais como dosagem, eficácia e segurança da composição. Na fase clínica III são realizados testes em um número bastante significativo de pacientes (1.000-3.000 indivíduos), distribuídos amplamente em regiões geográficas representativas, para uma melhor avaliação das propriedades farmacocinéticas, além da eficácia e segurança. Ao término da fase III, todos os dados clínicos obtidos são organizados e submetidos à agência reguladora (e.g., FDA, ANVISA) para solicitação da aprovação de um novo fármaco (NDA, da sigla inglesa para new drug application). Após a aprovação da NDA, o novo medicamento é lançado no mercado farmacêutico. Na fase clínica IV, 
é realizado o monitoramento do fármaco pós-comercialização (i.e., farmacovigilância), a longo prazo. ${ }^{14,15}$

\section{CONSIDERAÇÕES FINAIS}

A necessidade de novas alternativas terapêuticas para as DTNs é clara, mas os investimentos globais em P\&D ainda são extremamente insuficientes. É importante incentivar o estabelecimento de mecanismos apropriados para intensificar os investimentos em infraestrutura e qualificação de pessoal, dentro de uma visão estratégica que contemple a continuidade. $\mathrm{O}$ estabelecimento de redes globais tem se fortalecido como paradigma moderno essencial para uma mudança neste panorama. Nosso grupo de pesquisa tem contribuído de forma expressiva para esta área, consolidando novas fronteiras e incorporando elementos como inovação e integração. A importância do tema e o seu apelo mundial, aliados as interfaces multi-, inter- e transdisciplinares que conectam a Química a esta área fundamental da saúde pública do país e do mundo, são eixos motivadores para enfrentarmos com coragem e determinação os desafios científicos e tecnológicos do presente e do futuro.

\section{AGRADECIMENTOS}

Os autores agradecem à FAPESP (Fundação de Amparo à Pesquisa do Estado de São Paulo), CNPq (Conselho Nacional de Desenvolvimento Científico e Tecnológico), CAPES (Coordenação de Aperfeiçoamento de Pessoal de Nível Superior), OMS (Organização Mundial da Saúde), DNDi (Iniciativa Medicamentos para Doenças Negligenciadas) e MMV (Medicamentos para Malária) pelo contínuo apoio a pesquisa, desenvolvimento e inovação na área de fármacos para DTNs no Brasil. À Sociedade Brasileira de Química e, em especial, aos membros da Comissão do "Censo da Química no País" pela oportunidade de apresentar esta contribuição.

\section{REFERÊNCIAS}

1. Pinto, A. C.; Zucco, C.; Galembeck, F.; Andrade, J. B.; Vieira, P. C.; Quim. Nova 2012, 35, 2092.

2. http://www.who.int/neglected_diseases/2010report/WHO_NTD_report_update_2011.pdf, acessada em Setembro de 2013.

3. Accelerating Work to Overcome the Global Impact of Neglected Tropical Diseases: A Roadmap for Implementation. World Health Organization, 2012.

4. Adams, J.; Gurney, K. A.; Pendlebury, D.; Thomson Reuters Global Research Report, Neglected Tropical Diseases. Thomson Reuters, 2012.

5. Nwaka, S.; Ramirez, B.; Brun, R.; Maes, L.; Douglas, F.; Ridley, R.; PLoS Negl. Trop. Dis. 2009, 3, e440.

6. http://www.doctorswithoutborders.org/events/symposiums/2012-lives-in-the-balance/assets/files/Medical-Innovations-for-Neglected-Patients. pdf, acessada em Setembro de 2013.

7. Lombardino, J. G.; Lowe III, J. A.; Nat. Rev. Drug Discovery 2004, 3, 853.

8. McGee, P.; Drug Discovery Dev. 2006, 9, 16.

9. Anders, H. J.; Vielhauer, V.; Drug Discovery Today 2007, 12, 446.

10. Guido, R. V. C.; Oliva, G.; Andricopulo, A. D.; Pure Appl. Chem. 2012, 84, 1857.

11. Kogej, T.; Engkvist, O.; Blomberg, N.; Muresan, S.; J. Chem. Inf. Model. 2006, 46, 1201

12. Balakin, K. V.; Tkachenko, S. E.; Kiselyov, A. S.; Savchuk, N. P.; Drug Discov. Today: Technol. 2006, 3, 397.

13. Kitchen, D. B.; Decornez, H.; Furr, J. R.; Bajorath, J.; Nat. Rev. Drug Discovery 2004, 3, 935.

14. Dimasi, J. A.; Hansen, R. W.; Grabowski, H. G.; J. Health Econom. 2003, 22, 151 .

15. Nwaka, S.; Ridley, R. G.; Nat. Rev. Drug Discovery 2003, $2,919$. 